\title{
DesarRollo de LA COMPETENCIA LECTORA DE TEXTOS CIENTÍFICOS EN TRES ETAPAS DE FORMACIÓN UNIVERSITARIA. ESTUDIO DE CAMPO
}

\author{
Iraís Hernández Suárez \\ Bernardo Riffo
}

Resumen: Este artículo presenta los resultados de una investigación de campo, cuyo propósito central es dar cuenta del proceso de transformación de estudiantes universitarios de biología de la Universidad Veracruzana en Veracruz, México, en el desarrollo de habilidades para leer textos académicos disciplinarios. El estudio empírico consiste en un análisis de los resúmenes producto de la lectura de tres textos (artículo científico, libro de texto y manual de prácticas de laboratorio) escritos por jóvenes de tres niveles de formación (principiantes, intermedios y avanzados). Los resultados muestran una clara tendencia, a medida que avanzan en la formación universitaria, hacia el desarrollo de habilidades específicas para la lectura de textos especializados, las que incluyen el uso de estrategias para el adecuado manejo de los géneros discursivos propios de la comunidad académica de biología y suponen, además, la aplicación de conocimiento especializado en la disciplina.

Palabras clave: comprensión de textos académicos, desarrollo de habilidades de lectura, género discursivo, discurso especializado.

Enviado a dictamen: 17 de junio de 2009

Aprobación: 2l de septiembre de 2009

Revisiones: 1

Iraís Hernández Suárez, doctorante en Lingüística por la Universidad de Concep ción, profesora de tiempo completo de la Universidad Veracruzana, Correos electrónicos: irhernandez@uv.mx; irhernandez@udec.cl; iraisuv@prodigy.net.mx. Bernardo Riffo, doctor en Lingüística por la Universidad de Bielefeld (Alemania), Profesor Asociado del Departamento de Español, Facultad de Humanidades y Arte de la Universidad de Concepción, Chile. Correo electrónico: bernardo@udec.cl.
Abstract: This article presents the results of a field research study whose main purpose is to give an account of the transformation process university students of biology at Universidad Veracruzana (México) go through in developing their academic reading abilities in their discipline. The empirical study consisted of an analysis of the summaries written by students at three disciplinary levels (elementary, intermediate and advanced), produced when reading three texts (a scientific article, a textbook and a laboratory procedures manual). Results show a clear tendency to parallel progress in the discipline with development of specific abilities for reading specialized texts, which include both application of specialized knowledge of the discipline and strategies for the appropriate use of discursive genres pertaining to the academic community of biology.

Key words: academic reading, development of reading abilities, discourse genre, specialized discourse.

\section{Introducción}

T a Universidad Veracruzana es una de las instituciones públicas de educación superior más importantes de México, ocupa el quinto lugar en términos de la población estudiantil que atiende y de otros indicadores, como el número y la calidad de programas educativos que ofrece. Por ubicarse geográ- 
ficamente en la zona sureste del país, que cuenta con alta densidad poblacional, existe una gran demanda de ingreso y, por ende, un alto índice de rechazo, dada la insuficiente capacidad para atenderla. Esta situación justifica desde hace un par de décadas que el proceso de ingreso esté precedido por una rigurosa selección.

El examen para el ingreso, elaborado por el Centro Nacional de Evaluación (CENEVAL), incluye una sección que evalúa la habilidad verbal. Entre otros aspectos, se mide la comprensión de textos mediante una serie de ítems. Al igual que otras instituciones educativas públicas de México, la Universidad Veracruzana -en respuesta a la preocupación por incrementar la eficiencia de los universitarios en la lectura - integra en su currículum un Taller de Lectura y Redacción a través de textos contemporáneos. Este curso se imparte dentro de todos los programas educativos de las cinco áreas de conocimiento: técnica, ciencias de la salud, económicoadministrativas, humanidades y artes.

Con la finalidad de ofrecer capacitación a los docentes que deben impartir este curso, la Universidad Veracruzana implementó un Diplomadoen competencias de la lengua española; quienes coordinan, integran los contenidos e imparten este diplomado son preferentemente profesores de Literatura. Existe además un programa de fomento de la lectura (Programa Permanente de Formación de Lectores), que se propone como objetivo acercar a los universitarios a la literatura, incrementando el número de lectores y de lecturas, el cual realiza varias actividades. Como parte de este programa se ha editado una colección de textos bajo el cuidado del reconocido escritor Sergio Pitol, quien selecciona, edita y prologa las obras publicadas. ${ }^{1}$

La preocupación por el número de textos que en promedio se leen por año tiene su origen en las estadísticas publicadas en 2006 por el Consejo Nacional para la Cultura y las Artes (CONACULTA) mediante la Encuesta Nacional sobre Lectura (ENL). Los resultados indicaron como dato más destacado que los mexicanos leen en promedio 2.9 libros al año, cuando en los países desarrollados el promedio es de 15 libros por persona. En este contexto, otra acción dentro del programa de formación de lectores fue la aplicación de una encuesta sobre lectura. Una encuesta análoga a la ENL se realizó durante 2007 en la Universidad Veracruzana con la finalidad de conocer los hábitos de lectura de sus integrantes: estudiantes, profesores, trabajadores administrativos y manuales. Se indagó acerca del número de libros leídos en el último año, las preferencias de lectura, los horarios y lugares frecuentados para ello, entre otros datos que integraron lo que puede denominarse como una sociología de la lectura universitaria.

Entre los resultados más interesantes se subraya que 95\% de los universitarios dice haber leído menos de 10 libros durante el año anterior; las áreas académicas de artes y de humanidades alcanzaron los porcentajes más altos, en tanto que las áreas técnica y biológica-agropecuaria obtuvieron los porcentajes más bajos. El rango de mayor edad reportó un índice más alto de lectura. El área biológico-agropecuaria registró el mayor índice de lectura de revistas (88\%), siendo más representativas las revistas científicas (56\%).

A pesar de contar con esta información estadística general en torno a la lectura universitaria, se desconocen de manera específica las estrategias que los lectores individuales emplean cuando enfrentan cotidianamente la lectura de textos de su área de conocimiento disciplinar. Por tanto, se requiere no sólo conocer los hábitos de lectura de los universitarios, sino describir el procesamiento de lectura seguido por ellos cuando enfrentan textos académicos.

\section{Fundamentación teórica}

Desde la psicolingüística cognitiva se han propuesto modelos de comprensión de textos (Van Dijk y Kintsch, 1983; Kintsch, 1998) que permiten un acercamiento a este proceso. Gran parte de la investigación se ha aplicado a la lectura de texto narrativo en condiciones experimentales, sin embargo, existe poca investigación acerca 
de la comprensión de texto científico por parte de los investigadores del discurso. (Otero, León y Graesser, 2002: xi); la aplicación del modelo teórico propuesto por Kintsch (1998) para dar cuenta de la lectura de texto especializado de carácter expositivo entre universitarios es una contribución a esta ausencia.

Por otra parte, desde la perspectiva de la teoría del género que a partir de Swales (1990) ha generado una prolífica línea de investigación, existe una fuerte tendencia por reconocer la necesidad de abordar la lectura del texto especializado, en virtud del reconocimiento de la especificidad de los textos académicos. En particular, se enfatiza el estudio del discurso científico especializado en términos de su caracterización; Tardy $\&$ Swales (2008) presentan un panorama evaluativo sobre la investigación de las propiedades organizativas de los textos escritos, entre las que se ubican los estudios del género. Consideran que hay cuatro áreas que han recibido atención por parte de muchos investigadores: a) una dedicada a la estructura discursiva; b) otra que da cuenta de la forma genérica en relación con las orientaciones sociales: c) estudios dedicados a la coherencia y cohesión textuales y d) por último, aquéllos que se han dedicado al análisis del corpus y patrones fraseológicos.

Cuando los estudiantes ingresan a la educación universitaria, el largo proceso que han seguido a través de la formación básica y media ha permitido su incorporación en la sociedad letrada, de manera más o menos aceptable. No obstante, a partir de este momento, su proceso de inserción en una comunidad discursiva académica recién se inicia y, con ello, el proceso de alfabetización académica, entendido éste como el conjunto de nociones y estrategias necesarias para participar en la cultura discursiva de las disciplinas, así como en las actividades de producción y análisis de textos requeridas para aprender en la universidad, las que se adquieren precisamente en este proceso de inserción en la comunidad discursiva (Carlino, 2005: 13).

Este proceso se realiza de manera inconsciente, en virtud de que no existe un entrenamiento explíci- to dirigido a adquirir las estrategias necesarias en la producción y análisis de textos académicos, por lo que éstas se desarrollan a lo largo del proceso estudiantil de inmersión en la comunidad académica mediante el contacto continuo con los materiales de lectura.

Según el área disciplinaria de que se trate, los miembros expertos que conforman cada comunidad discursiva específica producen y consumen de manera habitual un conjunto de textos académicos y profesionales, lo que constituye géneros específicos. En cada ámbito académico, los textos presentan ciertas características que para los lectores expertos -individuos ya formados en la disciplina e integrados plenamente en la comunidad discursiva - resultan familiares, pero no necesariamente para el lector que recién inicia este proceso, de modo que el universitario asume la lectura de este tipo de textos en forma cotidiana como parte de su proceso de aprendizaje y estrechamente vinculado con él. Cada estudiante, a partir de su conocimiento y experiencia previos, desarrolla las estrategias de comprensión de los textos académicos con los que tiene contacto a lo largo de su formación universitaria, considerando las particularidades de cada subgénero académico.

Se entiende por género académico una clase de eventos comunicativos que obedecen a propósitos comunicativos compartidos por los miembros de la comunidad participantes en el proceso de interacción discursiva. Tales propósitos, que constituyen un aspecto central del género, son reconocidos por los miembros expertos. Esta característica fundamental le da forma a la estructura esquemática del discurso y restringe la selección del contenido. Además del propósito, los ejemplares de un género muestran similitudes en cuanto al contenido y la audiencia a la que van dirigidos los discursos (audiencia intencional). Cuando un discurso cumple con todos estos requisitos se considera un ejemplar prototípico de ese género (Swales, 1990: 58). Los géneros académicos, científicos y profesionales se caracterizan por el texto expositivo. La mayor parte de los estudios sobre comprensión lectora, no obstante, han tenido como objeto 
el texto narrativo. Recientemente, se ha señalado la necesidad de abordar el análisis de la comprensión de texto expositivo y, específicamente, abordar la lectura de textos científicos académicos.

Dentro de los ejemplares prototípicos, en el ámbito educativo se cita el libro de texto, en tanto que el artículo científico constituye el prototipo de los géneros que circulan en el espacio profesional propiamente. En ciertas áreas de conocimiento temático, como el de la biología, también tiene presencia el manual de prácticas de laboratorio. Aunque está dirigido intencionalmente a los profesionales, la audiencia incidental del artículo científico es también la de los estudiantes, quienes aspiran a formar parte de la comunidad científica profesional (Goldmann y Bizans, 2002: 39).

En este contexto, cuando se propone estudiar el fenómeno de la comprensión de textos académicos especializados como parte de un proceso sociocultural que implica la incorporación de un individuo en una comunidad discursiva específica, se hace necesario contar con fundamentos teóricos de tres fuentes, a saber: a) una que provea los conceptos y herramientas para estudiar el discurso como género, b) otra que proporcione los fundamentos psicolingüísticos para explicar el proceso mental de la lectura, y c) una que permita observar el fenómeno como un proceso cultural que involucra al lector y la comunidad en la que la lectura tiene lugar.

La teoría del género permite identificar las características textuales de los distintos géneros académicos y también permite considerar el propósito de la lectura (como característica genérica) que guía el proceso de comprensión. Dentro de las características estructurales, además de la organización global del texto, se consideran las numerosas señales, como los títulos y subtítulos, las marcas tipográficas, las oraciones temáticas, los marcadores discursivos, las marcas sintácticas, semánticas y paratextuales. Igualmente debe considerarse el papel de las estructuras retóricas o esquemas textuales que permiten al lector reconocer cómo está organizado el texto (descripción, clasificación, enu- meración, comparación y contraste). En este marco, se concibe la lectura como una lectura situada, que persigue un propósito específico y que se ejecuta en determinado contexto sociocultural, lo que posibilita la explicación del conocimiento previo como un factor fundamental en el proceso de comprensión.

La psicolingüística, a su vez, plantea que durante la lectura el sujeto construye una representación mental del texto. Para Kintsch (1998: 50), el modelo de situación constituye el nivel de representación de lo referido por el texto, al que preceden el nivel de representación de base textual (representación semántica) y el de superficie textual (representación de la forma sintáctica y léxica). Cuando se alcanza el modelo de situación, el lector es capaz de reproducir, luego, el contenido del texto sin necesidad de recurrir a la expresión textual. En el nivel más elemental, el de la superficie del texto, el lector dispone de la información que le permite reproducir sólo la forma superficial, la expresión de éste, sin que exista necesariamente comprensión.

En la construcción del modelo de situación, el lector hace acopio de sus conocimientos previos para integrarlos con la información que provee el texto, de manera que el resultado sea coherente y en ocasiones más rico. Los conocimientos previos incluyen conocimiento de contenido o temático, conocimiento de la estructura general del discurso y conocimiento específico de las estructuras del discurso usadas en determinado dominio. En el caso de la comprensión de textos científicos, varias clases de conocimiento previo son potencialmente relevantes para construir una representación (Goldmann y Bizans, 2002:20). También el conocimiento previo hace referencia al contexto sociocultural y las reglas de producción y circulación de los discursos, entre ellas, el conocimiento específico del propósito comunicativo de cada género discursivo, las características que lo constituyen y el rol de los participantes en el intercambio discursivo; es decir, un conocimiento situacional-interaccional.

Un enfoque sobre el género que asume el procesamiento del discurso como un proceso multidimensional 
con aspectos tanto cognitivos como socio-comunicativos, considera también los usos y propósitos comunicativos, además de las características formales de los textos. Se requiere, por tanto, dar cuenta de un conocimiento previo abarcador - multidimensional - tal como lo expone en extenso Johns (1997). En una propuesta cognitivo-comunicacional, el saber sobre los géneros es multidimensional (una competencia genérica), ya que comprende cualidades prototípicas referidas a las distintas dimensiones de los textos, lo que pone en juego "un conjunto de esquemas de operaciones cognitivas, variados sistemas de conocimiento interrelacionados: enciclopédico (sobre el mundo), lingüístico (léxico y gramática), interaccional-situacional y conocimiento sobre clases de textos" (Ciapuscio, 2005: 45).

En este punto es necesario advertir que el concepto de género está lejos de considerar la posibilidad de establecer parámetros universales, sino que, por el contrario, apunta la necesidad de plantear valores culturales en los cuales se considera como 'cultural' lo que procede de una comunidad discursiva determinada y no de nacionalidades. Por todo lo anterior, es necesario adoptar un enfoque etnográfico que permita acceder a las comunidades discursivas, tal como un antropólogo asume su inserción en una comunidad ajena, cuando se sigue el proceso de incorporación en una comunidad disciplinaria para analizar las estrategias usadas en el procesamiento textual por parte de sus miembros.

\section{El estudio}

La presente investigación aborda el proceso de comprensión de texto expositivo en el ámbito académico dentro del área de conocimiento de la biología. Específicamente, se requiere conocer de manera puntual cómo se da este proceso en el caso específico de los estudiantes que cursan y egresan de la carrera de biología en la Universidad Veracruzana y más precisamente, cómo se produce la modificación de sus conductas lectoras como evidencia de una incorporación progresiva en la comu- nidad discursiva académica. Las preguntas iniciales de investigación son: a) icómo se procesa la información de textos académicos por parte de los lectores del área disciplinaria de biología? y b) iqué cambios se pueden observar comparativamente en el desempeño lector de los alumnos principiantes, intermedios y avanzados?

Los datos corresponden al análisis del resumen resultante de la lectura de tres textos representativos de tres subgéneros académicos tomando en consideración el criterio de eficacia en el resumen y las estrategias usadas por el lector para establecer qué representación del texto construye el lector cuando lee textos académicos. Se considera resumen un texto derivado de otro, producido por reducción, que rescata lo más relevante desde el punto de vista del lector y su propósito de lectura, debe ser equivalente al texto fuente en términos informativos pero tener autonomía respecto de él (Carlino, 2005: 83). La representación textual es una mezcla del texto base y el modelo de situación, en éste se expresa la organización y estructura retórica del texto, además del conocimiento previo del lector.

En la elaboración del resumen, el peso otorgado al texto base o al modelo de situación depende del conocimiento previo que el lector posee para recuperar la información temática pertinente y su habilidad para ligarla de manera coherente. Por lo que un lector con escaso conocimiento previo - tanto temático como lingüístico o discursivo - ajusta su resumen al texto base, de tal manera que hace una reproducción textual o casi textual del texto que ha leído. En la medida en que el lector posee un conocimiento más amplio y abarcador, su resumen de lectura parte de una representación mental basada en un modelo de situación, que integra de manera coherente ideas de diferentes partes del texto, sin seguir necesariamente una paráfrasis del mismo, sino generando una macro estructura adecuada.

El objetivo de este estudio fue describir y caracterizar el desempeño lector de textos especializados por parte de estudiantes de biología en las etapas inicial, intermedia y avanzada a través del análisis del resu- 
men elaborado por quienes conformaron la muestra. Se manejó como hipótesis que: a) El texto científico universitario utilizado para la enseñanza de la biología posee características tales que su comprensión requiere, por parte de los lectores, la adquisición de conocimientos específicos, propios de la comunidad discursiva científica; b) El lector universitario transforma el conocimiento nuevo en conocimiento dado en su proceso de endoculturación al interior de la comunidad científica universitaria; y c) El lector universitario desarrolla paulatinamente estrategias lectoras -incrementa su capacidad inferencial - en la medida en que se incorpora en la comunidad discursiva científica.

Esta investigación además de descriptiva, fue exploratoria y de campo: descriptiva en tanto da cuenta, en términos cuantitativos y cualitativos, del incremento de las estrategias de lectura y del desarrollo de la capacidad inferencial de los estudiantes que ingresan, cursan y egresan de la carrera de biología en la Universidad Veracruzana. Por otra parte, dado que no existían datos previos en relación con la temática específica de esta investigación, que detallaran las características del lector universitario, y del desarrollo de sus estrategias de lectura, se consideró la necesidad de realizar un sondeo que permitiera elaborar una descripción, cuyo resultado se concibiera como la configuración del lector universitario. Por último, se reunieron condiciones para la realización de una investigación de campo, en tanto que la obtención de datos se llevó a cabo in situ, en el espacio propio de los sujetos en estudio. A la manera de las investigaciones de corte etnográfico, se ingresó en la comunidad discursiva de estudio para interactuar con los integrantes de esa comunidad empleando las técnicas de trabajo de campo.

Gracias al trabajo de ingreso en la comunidad discursiva objeto de estudio fue posible obtener información acerca de los textos que circulan habitualmente en el medio, a saber, el espacio académico de biología en la Universidad Veracruzana. Así, se eligieron tres subgéne- $\operatorname{ros}^{2}$ y se trabajó con ellos preparándolos con la finalidad de obtener resúmenes de un conjunto de lectores. Los subgéneros seleccionados fueron:

a. El artículo cientifico: es un texto de 5 mil 46 palabras, cuya temática versa sobre la dispersión de semillas por los murciélagos y su efectoen la regeneración de bosques y selvas, con gráficos y tablas que condensan datos, así como los característicos subtítulos y marcas tipográficas que organizan la estructura de un artículo: resumen, palabras clave, introducción, el desarrollo, discusión y bibliografía.

b. Ellibrodetexto: es una sección del capítulo 50 sobre Ecología, tomado del libro Biología de Campbell y Reece, que consta de 2 mil 253 palabras, un texto multimodal, con gráficos, recuadros, fotografías y subtítulos con distinta tipografía.

c. El manual de prácticas de laboratorio: es una práctica que aborda la diversidad celular que sólo posee 568 palabras y una estructura global convencional organizada por subtítulos: objetivo, introducción, materiales, método y resultados.

El estudio se aplicó a 42 sujetos, constituyendo una muestra no probabilística, por accesibilidad, por cuotas y voluntaria. Los participantes respondieron a la invitación, una vez que fueron seleccionados, cuidando que conformaran una distribución por cuotas cubriendo tres niveles de inserción en la comunidad discursiva: iniciales, intermedios y avanzados; de modo que 16 correspondían al nivel inicial y 13 a cada uno de los restantes. Los sujetos del nivel inicial cursaban los primeros 200 créditos de la carrera, los intermedios superaban los 200 pero aún no se encontraban en la etapa terminal de la carrera, en tanto, los avanzados se encontraban en la fase de elaboración de tesis (etapa terminal), realizando trámites para ingresar o cursando ya el postgrado. Se cuidó además que la muestra estuviera equilibrada tanto en el número de hombres y mujeres como en los promedios de calificación obtenidos. 
La interacción con los lectores se efectuó de manera personal, directa y cara a cara para realizar la entrevista y presentar cada texto, del cual se solicitó la elaboración del resumen. La instrucción para elaborar el resumen indicó "que lo hiciera como si tratara de trasmitir lo más relevante a un compañero".

Se presentaron los materiales de lectura de tres subgéneros ya mencionados: un artículo de investigación, un apartado o sección de un capítulo de un libro de texto y una práctica de laboratorio integrada en un manual, a los que se ha denominado texto fuente. ${ }^{3}$ Cada sujeto leyó cada uno de los tres subgéneros en el orden de su preferencia e inmediatamente procedió a resumirlo. Se obtuvieron así 42 resúmenes por cada subgénero, y el corpus total a analizar quedó constituido por 126 resúmenes.

Para establecer adecuadamente los criterios de análisis del resumen fue necesario conocer en detalle las características de los textos fuente, por lo que, una primera tarea - en términos metodológicos- consistió en la identificación de las ideas consideradas relevantes desde el punto de vista del texto, es decir, las unidades idea (UI) en el texto fuente. Se utilizó el concepto de modelo de situación, de manera que permitiera reconocer en el texto los elementos constituyentes de la situación: ¿quién hace qué?, ¿dónde?, ¿cuándo?, ¿cómo? y ipara qué? De esta manera, además de clasificar cada sección del texto de acuerdo al elemento correspondiente, se recuperó una meta enunciado resultante de enfocar su contenido desde la perspectiva del modelo de situación. Se constituyó así un parámetro que permitió contrastar la ejecución del resumen con la estructura y el contenido del texto fuente.

A partir de estudios análogos se elaboraron los criterios de análisis del resumen, se recuperó el criterio de la eficacia en el resumen, considerado como la proporción entre el número de ideas juzgadas importantes incluidas y el número total de palabras en cada resumen (Garner, 1982: 275). Otro estudio más, que aborda el proceso de comprensión de textos entre estudiantes universitarios, fue tomado en cuenta, el de Johns \& Mayes (1990) quienes propusieron como criterio la clasificación de las estrategias ${ }^{4}$ en la elaboración de los resúmenes, siguiendo el modelo de situación propuesto desde Van Dijk y Kintsch (1978), según el cual, los resúmenes que mantienen la estructura de superficie del texto fuente son producto de las estrategias más elementales: la copia transcripción y la paráfrasis. En tanto, otras estrategias como la construcción de macro proposiciones de un parágrafo o de una selección parten de la representación de base textual; por último, la elaboración de un meta enunciado implica la construcción de una representación del modelo de situación. En contrapartida, se localizó la producción de distorsiones del texto fuente (Johns $\&$ Mayes, 1990: 258).

\section{Resultados}

Si se considera que el número de palabras del texto fuente en cada uno de los géneros académicos utilizados era considerablemente diferente, la reducción en el resumen de cada uno de ellos no se refleja en la misma proporción. Podría esperarse como resultado que la reducción del texto en el resumen coincidiera con la disminución en la extensión del texto fuente.

Los sujetos del nivel avanzado respondieron a esta expectativa, ya que los promedios del número de palabras fueron en orden decreciente: 138, 115 y 58; artículo, libro y manual, respectivamente. Aunque los novatos también presentaron decremento en el número de palabras, éste no destaca tanto: ya que sólo pasó desde 145 hasta 132 y hasta 100. Por el contrario, los sujetos del nivel intermedio incrementaron el número de palabras del resumen del libro de texto, aunque mantuvieron el decremento del resumen de la práctica.

Estos resultados tuvieron su impacto en el índice de eficacia, ya que en cada caso las ideas relevantes, organizadas de acuerdo con el modelo de situación, sumaron cinco en cada uno de los textos-fuente. El 
índice de eficacia ${ }^{5}$ mostró la tendencia general al incremento por parte del nivel avanzado (menor número de palabras para expresar un mayor número de ideas), que alcanzó diferencias claramente establecidas con los otros niveles en el caso del manual de laboratorio y del artículo científico.

Tabla 1. Índice de eficacia en el resumen del artículo

\begin{tabular}{|l||c|c|c|}
\hline \multicolumn{1}{|c|}{ Artículo } & $\mathbf{N}^{\circ}$ palabras & $\mathbf{N}^{\circ}$ ideas & $\begin{array}{c}\text { Índice de } \\
\text { Eficacia }\end{array}$ \\
\hline \hline Iniciales & 145 & 3.68 & 42.10 \\
\hline \hline Intermedios & 107 & 3.00 & 40.91 \\
\hline \hline Avanzados & 138 & 4.07 & 32.79 \\
\hline
\end{tabular}

En el manual de laboratorio, la diferencia entre iniciales y avanzados fue de 33.08 que indica cuánto pueden alcanzar en eficacia los lectores que pertenecen a este sector al resumir un texto del subgénero práctica de laboratorio. En el artículo, los lectores del nivel avanzado aventajaron por casi diez puntos (9.31) a los lectores iniciales. En cuanto al libro de texto, el índice de eficacia se encontró muy cercano entre los tres sectores y fue el nivel inicial el que alcanzó mejor resultado.

Tabla 2. Índice de eficacia en el manual de laboratorio

\begin{tabular}{||c|c|c|c||}
\hline $\begin{array}{c}\text { Manual } \\
\text { laboratorio }\end{array}$ & $\begin{array}{c}\mathbf{N}^{\circ} \text { de } \\
\text { palabras }\end{array}$ & $\begin{array}{c}\mathbf{N}^{\circ} \text { de } \\
\text { ideas }\end{array}$ & $\begin{array}{c}\text { Índice de } \\
\text { eficacia }\end{array}$ \\
\hline \hline Nivel inicial & 110.8 & 2.31 & 57.33 \\
\hline \hline Nivel intermedio & 59.8 & 2.30 & 31.09 \\
\hline \hline Nivel avanzado & 58.8 & 2.46 & 24.25 \\
\hline
\end{tabular}

Tabla 3. Índice de eficacia en el libro de texto

\begin{tabular}{||c|c|c|c||}
\hline Libro de texto & $\mathbf{N}^{\circ}$ palabras & $\begin{array}{c}\mathbf{N}^{\circ} \text { de } \\
\text { ideas }\end{array}$ & $\begin{array}{c}\text { Índice de } \\
\text { eficacia }\end{array}$ \\
\hline \hline Nivel inicial & 118.5 & 3.4 & 32.97 \\
\hline \hline Nivel intermedio & 138.7 & 3.4 & 39.23 \\
\hline \hline Nivel avanzado & 120.8 & 3.3 & 35.08 \\
\hline
\end{tabular}

a) Artículo científico. Cuando los lectores leyeron y resumieron el artículo científico, la frecuencia de copia transcripción, la más elemental de las estrategias, fue usada por el nivel inicial en $12.5 \%$ y por el nivel intermedio en $46 \%$, mientras que el nivel avanzado no hizo uso de ésta. Las paráfrasis, estrategia muy cercana a la copia transcripción, fue mayoritariamente usada por los sujetos del nivel inicial (5\%), un poco menos en los intermedios (3\%) y se encontró ausente en los avanzados. La combinación, estrategia que consiste en recuperar ideas de distintos parágrafos y combinarlas, fue usada de manera exclusiva por los del nivel intermedio, en 9\%. Las macro proposiciones pueden ser de dos tipos: como generalización de un parágrafo o como generalización de una selección; en ambos casos se elabora una representación más compleja del texto. Esta alternativa fue usada en el artículo por miembros de nivel inicial (siete generalizan el párrafo y once generalizan una selección) y del nivel intermedio, tres de cada uno. Por último, los lectores de nivel avanzado, cuando elaboraron resumen del artículo, recurrieron a la construcción de meta enunciado en $85 \%$, frente a $23 \%$ de los intermedios y $12.5 \%$ de los iniciales.

\section{Tabla 4. Porcentaje de algunas estrategias usadas en el} resumen del artículo científico

\begin{tabular}{|l|l|l|l|}
\hline & \multicolumn{1}{|c|}{$\begin{array}{c}\text { Copia } \\
\text { transcripción }\end{array}$} & Paráfrasis & $\begin{array}{c}\text { Meta } \\
\text { enunciados }\end{array}$ \\
\hline \hline Iniciales & $12.5 \%$ & $5.5 \%$ & $12.5 \%$ \\
\hline \hline Intermedios & $46 \%$ & $3.5 \%$ & $23 \%$ \\
\hline \hline Avanzados & $0 \%$ & $0 \%$ & $85 \%$ \\
\hline
\end{tabular}

b) Libro de texto. En el libro de texto, el resumen presentó copias transcripciones en lectores iniciales e intermedios (19 y 31\%, respectivamente), pero no entre los avanzados. Las paráfrasis en el libro de texto se presentaron numéricamente en cuatro, seis y dos sujetos de cada uno de los niveles, respectivamente. Las combinaciones hicieron presencia en los resúmenes de 
iniciales e intermedios, aunque en un bajo porcentaje (uno y seis sujetos, respectivamente). Las macro proposiciones constituyeron en el libro de texto, un recurso utilizado por los tres sectores (5-4-1 sujetos) que usaron macro proposiciones de generalización de un parágrafo. En tanto que siete sujetos del nivel inicial, seis del intermedio y dos del avanzado generalizaron las selecciones. En el libro de texto, los lectores avanzados mostraron su capacidad de elaborar meta enunciados en once casos, lo que representó $85 \%$, en tanto que los intermedios fueron tres sujetos (23\%), y fueron superados por los iniciales, donde cinco sujetos representaron $31 \%$ de aciertos en el meta enunciado.

Tabla 5. Porcentaje de algunas estrategias usadas en el resumen del libro de texto

\begin{tabular}{|l|l|l|l|}
\hline & $\begin{array}{c}\text { Copia trans- } \\
\text { cripción }\end{array}$ & Paráfrasis & $\begin{array}{l}\text { Meta enun- } \\
\text { ciados }\end{array}$ \\
\hline \hline Iniciales & $19 \%$ & $25 \%$ & $31 \%$ \\
\hline \hline Intermedios & $31 \%$ & $46 \%$ & $23 \%$ \\
\hline \hline Avanzados & $0 \%$ & $15 \%$ & $85 \%$ \\
\hline
\end{tabular}

c) Manual de prácticas. En el manual de laboratorio, el texto más reducido y organizado estructuralmente, la copia transcripción se presentó en menor porcentaje: $12.5 \%$ en los iniciales, $8 \%$ en los intermedios y estuvo ausente en los avanzados. Las paráfrasis del manual se presentaron en cuatro sujetos de nivel inicial, cinco del intermedio y ninguno en el avanzado. En cuanto a la combinación, hubo un solo caso entre los lectores del nivel intermedio. Las macro proposiciones fueron usadas en el manual únicamente por iniciales e intermedios; las generalizaciones del parágrafo en dos lectores iniciales y uno del nivel intermedio. Las generalizaciones de una selección en seis iniciales y tres intermedios. Finalmente, el manual obtuvo los resultados más altos en cuanto a la presencia de meta enunciados en los tres niveles, ya que los iniciales los elaboraron en 19\%, los intermedios en 31\% y los avanzados en $85 \%$ ( 5,5 y 12 sujetos, respectivamente).
Tabla 6. Porcentaje de algunas estrategias usadas en el resumen del manual de prácticas

\begin{tabular}{||l|c|c|c|}
\hline & $\begin{array}{c}\text { Copia } \\
\text { transcripción }\end{array}$ & Paráfrasis & $\begin{array}{c}\text { Meta } \\
\text { enunciados }\end{array}$ \\
\hline \hline Iniciales & $12.5 \%$ & $25 \%$ & $19 \%$ \\
\hline \hline Intermedios & $8 \%$ & $38 \%$ & $31 \%$ \\
\hline \hline Avanzados & $0 \%$ & $0 \%$ & $85 \%$ \\
\hline
\end{tabular}

En términos cuantitativos globales, las distorsiones fueron frecuentes en el resumen del artículo científico (14 sujetos), se redujeron en el libro de texto a siete y se presentaron sólo tres en el manual. Por cada uno de los niveles de inserción en la comunidad discursiva, la incidencia de sujetos que elaboraron distorsiones en el artículo se distribuye en seis iniciales, siete intermedios y uno de los avanzados. El libro de texto redujo la incidencia a tres sujetos en iniciales e intermedios y uno de los avanzados. El manual de laboratorio redujo aún más, ya que sólo hubo un sujeto que elaboró distorsiones por cada uno de los niveles.

\section{Discusión general}

La construcción de un modelo de situación, expresada mediante la elaboración del meta enunciado en el resumen, resulta de la información procedente del texto integrada con los conocimientos previos del lector (Kintsch, 1998). El modelo de situación se recupera a partir de las características básicas del texto como las marcas y subtítulos; pero requiere además la adecuada competencia genérica del lector que abarca el conocimiento temático, de la estructura textual y de la situación comunicativa (Johns, 2002; Goldmann y Bizans, 2002; Ciapuscio, 2005). No obstante, el desarrollo de estas estrategias es individual, se realiza sin guía por parte de los profesores, quienes se esfuerzan sólo por trasmitir el contenido propio de su área de conocimiento y se olvidan de la apropiación de las habilidades de lecto escritura de sus estudiantes, específicamente de textos académicos 
(Carlino, 2005: 165). Sin embargo, el adecuado manejo de estas habilidades y estrategias redunda en un mejor aprovechamiento en las asignaturas, en tanto que permite la comprensión y producción de textos de la manera que lo hacen quienes ya son miembros totalmente incorporados en la comunidad discursiva (Johns, 2002).

Autoras como Johns $(1997,2002)$ y Carlino (2005) señalan la necesidad de conducir este aprendizaje de manera simultánea a la adquisición del conocimiento temático, lo que se denomina literacidad académica o alfabetización académica. En la medida en que los lectores, al ingresar en una comunidad discursiva específica, se involucren con las prácticas discursivas habituales de manera guiada, es decir, conducida por los expertos ya incorporados en ella, la construcción de meta enunciados en cada género discursivo puede constituir también una práctica habitual. La adecuación del resumen construido por el lector estuvo en relación con identificar el género del texto fuente, reconocer el propósito tanto del texto fuente como del resumen que elaboró, manejar las estructuras, el estilo e incluso el léxico que supone un texto académico. Todo ello requería, además del conocimiento temático, un conocimiento discursivo específico de la comunidad de práctica.

Si bien el modelo de situación constituye la estrategia que permitió al lector elaborar meta-enunciados como resultado de su comprensión de una lectura, éstos se construyeron a partir del contenido informativo del texto fuente vinculado con los conocimientos previos del lector, entre los que se incluyó la competencia genérica. El análisis de las estrategias usadas por los lectores como resultado de su proceso de lectura, mostrado en los resúmenes, evidenció la tendencia al incremento por parte de los sujetos del nivel avanzado de estrategias más complejas, cuando se construyó el modelo de situación. Es decir, los lectores avanzados fueron más proclives a usar con mayor frecuencia la elaboración de meta enunciados en los tres subgéneros discursivos.

Por otro lado, el análisis de las distorsiones reveló parte de los conocimientos previos que se activaron en los lectores universitarios, porque en buena medida los casos constituyeron aportaciones al texto. Los otros tipos de distorsión fueron la contrapartida de las copias acertadas: pérdida o adición de información en la UI, combinaciones con ruptura, macro-proposiciones inexactas o meta enunciados inexactos.

El artículo científico propicióla presencia de diversas distorsiones, desde pérdidas y adiciones de información hasta la construcción de meta enunciados inexactos; las pocas distorsiones en el manual se debieron a imprecisiones o a macro proposiciones muy generales. El libro de texto propició en su mayoría distorsiones basadas en opiniones o juicios valorativos que el lector hizo sobre el texto, además de un par de rupturas en la combinación y una macro proposición inexacta.

La cantidad y calidad de las distorsiones en relación con un subgénero específico permitió, por un lado, evaluar la disímil dificultad de lectura entre un texto y otro (mayor en el artículo y menor en el manual) y, por otro, interpretar la distinta actitud asumida por parte del lector frente al texto. El libro de texto suscitó comentarios y juicios valorativos acerca del contenido, cuyo aporte muestra información de la ciencia ya establecida; en tanto que el manual, donde la información no requería mayor comentario, fue objeto de imprecisiones. El artículo, por su parte, dado lo extenso y complejo de su abordaje, abrió el abanico de distorsiones.

Así, cabe preguntarse por qué los lectores recurrieron a la copia transcripción y a la paráfrasis como estrategia para elaborar el resumen. Existen dos posibles respuestas, la primera es que el lector haya considerado una idea relevante procedente del texto fuente; es decir, la selección de macro proposiciones del texto fuente. La segunda posibilidad, es que el lector haya seleccionado una idea sin intentar reelaborarla de manera resumida, posiblemente a causa de las restricciones propias en sus habilidades de lectoescritura. Se debe advertir que la frecuencia de copia transcripción y de paráfrasis entre los iniciales redujo la posibilidad de una mayor presen-

Revista LiminaR. Estudios sociales y humanísticos, año 7, vol. VII, núm. 2, diciembre de 2009, Tuxtla Gutiérrez, Chiapas, México. ISSN: 1665-8027 127 
cia de distorsiones en este sector; ya que, el lector que recuperó el texto en su forma superficial necesariamente lo mantuvo sin alteración.

Por su parte, las distorsiones dejaron entrever el esfuerzo del lector por construir un modelo de situación, resultado de reunir la información proveniente del texto con sus conocimientos previos.

Los datos obtenidos en este estudio permiten considerar que los sujetos de nivel avanzado que recurrieron a la copia transcripción y a la paráfrasis, sólo lo hicieron en el caso del libro de texto, y no lo requirieron en el manual de laboratorio ni en el artículo científico. La descripción de hechos de la ciencia establecida (definiciones, entre otras), característica particular del libro de texto, condujo al lector - incluso al experimentado - a mantener la afirmación de la manera en que la presentaba el texto. Por tanto, la explicación del uso de esta aparentemente elemental estrategia, en los lectores avanzados cae en el primer caso, la usaron para recuperar hechos o afirmaciones ya establecidos en la ciencia.

Una explicación distinta se puede ofrecer en aquellos casos en que el lector sólo eligió las ideas que consideró relevantes y las reprodujo textualmente en su totalidad, como sucedió entre los lectores incipientes. En términos generales, se aprecia que los lectores superaron paulatinamente sus deficiencias lectoras de manera desigual y con altibajos, debido, posiblemente, a un disímil conocimiento previo y a la falta de guía por parte de los expertos.

En resumen:

a. Las diferencias obtenidas en el índice de eficacia entre lectores iniciales y avanzados evidenciaron la consciente reducción del texto fuente en el resumen y una acertada selección del contenido en términos de las UI expresadas en los lectores avanzados.

b. La copia transcripción constituyó un recurso muy utilizado por los lectores iniciales, en tanto que los avanzados prescindieron completamente de éste en el resumen del artículo científico y en el manual; su uso fue muy limitado en el libro de texto.

c. Se produjo una tendencia creciente en la presencia de meta enunciados en los resúmenes elaborados por los lectores avanzados.

d. Los lectores avanzados redujeron la cantidad de distorsiones en sus resúmenes de los tres subgéneros académicos.

e. Estos resultados constituyen evidencias de que los lectores que ingresaron en una comunidad discursiva especializada, como es en este caso la comunidad de biólogos, adquirieron paulatinamente habilidades estratégicas para leer textos especializados en su área de conocimiento. En la medida en que avanzaron en la formación académica, desarrollaron estrategias discursivas más complejas y eficaces para abordar los textos que formarán parte de su entorno profesional.

\section{Notas}

${ }^{1}$ Se puede consultar esta información en www.uv.mx/ lectores.

${ }^{2}$ En las referencias bibliográficas aparecen los datos de cada uno de los textos fuente.

${ }^{3}$ Texto fuente se denomina al texto que es leído por el sujeto y del cual parte la elaboración del resumen.

${ }^{4}$ Véase la tabla de clasificación de las estrategias en el cuadro anexo. Tabla 2.

${ }^{5}$ Un menor valor numérico indica mejor índice de eficacia.

\section{Bibliografía}

Campbell, Neil \& Reece, Jane (2007), "Introducción a la ecología y a la biosfera”, en Biología, Madrid: Médica Panamericana, pp. 1080-1083.

Carlino, Paula (2005), Escribir, leer y aprender en la Universidad. Una introducción a la alfabetización académica, Buenos Aires: F.C.E. 
Ciapuscio, Guiomar (2005), "La noción de género en la Lingüística Sistémico Funcional y en la Lingüística Textual", en Revista Signos, vol. 38, núm. 57, pp. 31-48.

Coutiño, Rocío y Fernández, Socorro (1997), "Diversidad celular", en Manual de Prácticas de laboratorio de Biología celular, Xalapa: Universidad Veracruzana, pp. 11-12.

Galindo, Jorge (1998), "Dispersión de semillas por murciélagos: su importancia en la conservación y regeneración del bosque tropical", en Acta Zoológica Mexicana, núm. 73, México, pp. 57-78.

Garner, Ruth (1982), "Efficient text summarization: costs and benefits", en Journal of Educational Research, may-june, vol. 75, núm. 5, Maryland: University of Maryland, pp. 274-279.

Goldman \& Bizans (2002), "Toward a functional analysis of scientific genres: Implications for understanding and learning processes", en José Otero, José A. León y Arthur C. Graesser (eds.), The Psychology of science texts comprehension, Mahwah, New Jersey: Lawrence Erlbaum Associates Publishers, pp. 19-50.
Johns, Ann \& Mayes, Patricia (1990), "An analysis of summary protocols of university ESL students", en Applied Linguistics, vol. 1l, núm. 3, San Diego: Oxford University Press, pp. 253-271.

Johns, Ann (1997), Text, role, and context: Developing academic literacies, Cambridge: Cambridge University Press.

Johns, Ann (Ed.) (2002), Genre in the classroom: multiple perspectives, Mahwah, New Jersey: Lawrence Erlbaum Associates Publishers.

Kintsch, Walter \& Van Dijk, Teun A. (1983), Strategies of Discourse Comprehension, New York: Academic Press.

Kintsch, Walter (1998), Comprehension: A paradigm for cognition, Cambridge: Cambridge University Press.

Swales, John (1990), Genre analysis: English in academic and research settings, Cambridge: Cambridge University Press.

Tardy, Christine \& Swales, John (2008), "Form, text organization, genre, coherence, and cohesion", en Charles Bazerman (Ed.), Handbook of research on writing: history, society, school, individual, text, New York: Erlbaum Associates, pp. 565-577. 
DesarRolLo de La COMPETENCIA LECTORA DE TEXTOS CIENTÍFICOS

\begin{tabular}{|c|c|c|c|}
\hline \multirow{2}{*}{ Características } & \multicolumn{3}{|c|}{ Sub-género académico } \\
\hline & Artículo científico & Libro de texto escolar & Manual de prácticas de laboratorio \\
\hline Propósito comunicativo & $\begin{array}{l}\text { Comunicar e informar los resultados de } \\
\text { un tema de investigación, dentro de un } \\
\text { campo específico, que se pone a consi- } \\
\text { deración de la comunidad profesional } \\
\text { científica de una determinada disciplina. }\end{array}$ & $\begin{array}{l}\text { Trasmitir información sobre contenidos } \\
\text { ya establecidos dentro de la comuni- } \\
\text { dad discursiva como parte del conoci- } \\
\text { miento disciplinar. }\end{array}$ & $\begin{array}{l}\text { Establecer las instrucciones que sirven } \\
\text { de guía para la realización de una prác- } \\
\text { tica de laboratorio. }\end{array}$ \\
\hline Roles participantes & $\begin{array}{l}\text { Presencia implícita del autor del artícu- } \\
\text { lo científico. } \\
\text { El autor supone una audiencia inten- } \\
\text { cional (la comunidad científica) a la } \\
\text { que no alude directamente. } \\
\text { Comunicación entre científicos. }\end{array}$ & $\begin{array}{l}\text { Ausencia de la voz de los autores del } \\
\text { libro de texto; el lector está implícito. } \\
\text { Información de los científicos a los } \\
\text { miembros que recién se incorporan a } \\
\text { la comunidad discursiva, mediada por } \\
\text { los editores del texto. }\end{array}$ & $\begin{array}{l}\text { Presencia implícita del autor del ma- } \\
\text { nual de prácticas. } \\
\text { El reconocimiento del lector mediante } \\
\text { la flexión verbal en imperativo de se- } \\
\text { gunda personal singular. }\end{array}$ \\
\hline Escenario o situación & $\begin{array}{l}\text { Comunidad profesional. } \\
\text { Los estudiantes constituyen una au- } \\
\text { diencia incidental. }\end{array}$ & $\begin{array}{l}\text { Educación formal. } \\
\text { Los estudiantes constituyen la audien- } \\
\text { cia intencional de manera implícita. }\end{array}$ & $\begin{array}{l}\text { Educación formal. } \\
\text { Los estudiantes constituyen la audien- } \\
\text { cia intencional de manera explícita. }\end{array}$ \\
\hline Estructura textual & $\begin{array}{l}\text { Introducción } \\
\text { Método } \\
\text { Resultados } \\
\text { Discusión } \\
\text { Referencias bibliográficas }\end{array}$ & $\begin{array}{l}\text { Índice general } \\
\text { Capítulos } \\
\text { Temas } \\
\text { Sub-temas } \\
\text { Conceptos clave } \\
\text { Desarrollo de conceptos } \\
\text { Figuras } \\
\text { Ejemplos } \\
\text { Referencias bibliográficas } \\
\text { (Texto multimodal) }\end{array}$ & $\begin{array}{l}\text { Objetivo } \\
\text { Introducción } \\
\text { Materiales y método } \\
\text { Resultados } \\
\text { Referencias bibliográficas }\end{array}$ \\
\hline
\end{tabular}

Tabla l. Características de los sub-géneros académicos

\begin{tabular}{|c|c|c|c|}
\hline \multicolumn{2}{|r|}{ Copias acertadas } & \multicolumn{2}{|r|}{ Distorsiones } \\
\hline \multirow{4}{*}{ 1. Reproducciones } & a. copia-transcripción & \multirow{4}{*}{ 1. Unidad idea } & a. pérdida \\
\hline & \multirow{3}{*}{ b. paráfrasis } & & b. adición \\
\hline & & & c. imprecisión en el S \\
\hline & & & d. imprecisión en el V \\
\hline \multirow{2}{*}{ 2. Combinaciones } & a. dentro del parágrafo & \multirow{2}{*}{ 2. Combinaciones } & a. parciales \\
\hline & b. a través del parágrafo & & b. ruptura \\
\hline \multirow{3}{*}{ 3. Macroproposiciones } & a. generalización del parágrafo & \multirow{3}{*}{ 3. Macroproposiciones } & a. enunciados muy generales \\
\hline & b. generalización de una selección & & b. macro-proposiciones inexactas \\
\hline & c. meta-enunciado & & c. meta-enunciados inexactos \\
\hline & & \multirow{4}{*}{ 4. Aportaciones } & a. comentarios sobre el texto \\
\hline & & & b. observaciones sobre la lectura \\
\hline & & & c. juicios de opinión \\
\hline & & & d. conclusiones participativas \\
\hline
\end{tabular}

Tabla 2. Cuadro Clasificatorio Johns \& Mayes, 1990. 